\title{
Respiratory muscle strength after upper abdominal surgery
}

The function of the respiratory system is invariably affected during and after abdominal surgery. ${ }^{1}$ This may be of no consequence in subjects with a large pulmonary reserve but may lead to postoperative pulmonary complications in high risk patients. Indeed, bronchoaspiration, atelectasis, pneumonia, and pulmonary oedema remain the most common causes of postoperative morbidity and a very important cause of mortality. Several studies have identified the factors that help predict the risk of development of postoperative pulmonary complications in these patients. ${ }^{23}$ The single most important one is the site of surgery. The closer an operation is to the diaphragm, the more likely is the chance of developing postoperative pulmonary complications. That is the reason why upper abdominal procedures are associated with a $20-40 \%$ incidence of postoperative pulmonary complications while lower abdominal surgery carries an incidence of $2-5 \% .{ }^{24}$ Other accepted risk factors include duration of surgery, age, obesity, smoking, sputum production, and history of lung disease. After upper abdominal operations all patients have decreased lung volumes and adopt a rapid shallow pattern of breathing. These changes resemble those seen in patients with neuromuscular disease and led some investigators to postulate that the mechanism for the genesis of postoperative pulmonary complications was best explained by diaphragmatic dysfunction. Subsequent studies in humans proved this to be the case. ${ }^{5}$ Patients undergoing cholecystectomy showed a decrease in transdiaphragmatic pressure and a shift to predominantly rib cage breathing for $24-48$ hours after surgery which gradually returned to normal. Studies in cholecystectomised dogs showed that neither the anaesthesia nor the pain were responsible for these findings, and that the best explanation is a decrease in its recruitment, secondary to an as yet unexplained reflex inhibitory mechanism. ${ }^{6}$ Recent data from studies on patients with an already compromised respiratory drive (sleep apnoea) showed that the administration of postoperative opiates further aggravated apnoea, tachyarrhythmias, and hypoxaemia. ${ }^{7}$ It is tempting to speculate that the opiates magnified the postoperative inhibitory reflex mechanism. If so, substances or manoeuvres that decrease this inhibition should have beneficial effects.

Theophylline shares many pharmacological properties with other naturally occurring alkaloids such as caffeine and theobromine. In general they are stimulants of the cardiovascular and central nervous systems, including the cortex, medulla and spinal cord. Specifically, theophylline increases the rate and depth of respiration. Because it is also a bronchodilator, it has enjoyed a significant role in the therapeutic armamentarium for the treatment of obstructive pulmonary disease. Unfortunately its therapeutic serum range is narrow and dangerously close to the toxic levels. Symptoms and signs of toxicity including severe convulsions may occur at levels not much higher than those clinically desired. This has led to an overall reassessment of the therapeutic role of theophylline. Several recent in vitro studies ${ }^{8}$ suggested that theophylline increased the force that diaphragmatic strips could generate at low stimulating frequencies (lower than 30 $\mathrm{Hz}$ ). The concentrations required for this effect were much higher than those achieved in vivo, so the question of the clinical relevance of these reports has remained a matter of debate. Studies on isolated diaphragmatic fibres which may more closely resemble the perfused state seen in patients have documented an $11 \%$ increase in the capacity to generate force at theophylline concentrations of $15 \mathrm{mg} / \mathrm{dl} .{ }^{9}$ Studies in normal volunteers and in patients have generated controversy, but it is fair to say that theophylline at clinically used levels has a minimal inotropic effect on the diaphragm. If, indeed, aminophylline increases diaphragmatic strength, it follows that an obvious question is whether aminophylline affects respiratory muscle strength after abdominal surgery and, if so, whether it may prevent the development of postoperative pulmonary complications.

In this issue of Thorax, Siafakas and coworkers (pages 693-7) report the results of a double blind randomised trial designed to evaluate the effect of aminophylline on respiratory muscle strength in patients undergoing cholecystectomy. As expected, all patients showed a decrease in forced vital capacity (FVC), forced expiratory volume in one second $\left(\mathrm{FEV}_{1}\right)$, maximal inspiratory (PImax) and expiratory (PEmax) pressures without changes in the FEV $V_{1} / F V C$ ratio. The drop in PImax was significantly smaller in patients who received aminophylline than in the patients who received placebo. In contrast, the drop in PEmax was similar in all treatment groups. The inescapable conclusion reached by the authors is that aminophylline had a protective effect only upon the inspiratory muscles, or that it acted centrally by reducing the inhibitory reflex. In their final sentence the authors suggest that aminophylline could be used to minimise complications after upper abdominal surgery. Before this recommendation is accepted, several results of the study must be discussed and an argument must be made to obtain more data. It is hard to reconcile the decrease of $50 \%$ in PImax and 25\% in PEmax in the untreated group with the lack of changes in FVC in these patients, since the volume expelled in this manoeuvre depends primarily on the force of the inspiratory and expiratory muscles. In other words, how can a weak pump not decrease the volume of expelled air? In this study we are not provided with the incidence of postoperative pulmonary complications in the population studied, even though the patients were closely followed for at least 48 hours. If the number of postoperative pulmonary complications was very low, why subject patients to a treatment with potential side effects when the benefit on the most important outcome, namely postoperative pulmonary complications, remains unproven? If indeed the basic mechanism in the genesis of postoperative pulmonary complications is a reflex inhibition of the diaphragm, primary stimulants of respiration may be even more beneficial. This seems to be supported by the study of Jansen $e t a l^{10}$ who showed that intravenous doxapram administered postoperatively resulted, not only in better oxygenation, but also in a decreased incidence of postoperative pulmonary complications. It is tempting to speculate that intermittent breathing of $\mathrm{CO}_{2}$ enriched gas mixtures would have the same beneficial effect. Finally, the advent of newer and less invasive surgical techniques such as laparoscopic surgery may result in decreases in the incidence of postoperative pulmonary complications to the point where any intervention may be saved only for those patients in the highest risk group. 
The use of a rational approach for the identification of patients at risk for development of postoperative pulmonary complications and their subsequent systematic study has led to our better understanding of the mechanisms responsible for their genesis. On the basis of these observations the rational testing of different therapeutic modalities has resulted in a progressive decrease in the incidence of postoperative pulmonary complications and their dire consequences. The true role of aminophylline in our armamentarium still remains to be determined, but it is refreshing to see that these questions are being approached in the best scientific spirit. Chief, Pulmonary Section Boston Veterans Medical Center, Massachusetts 02130 USA

Reprint requests to: Dr B Celli
1 Pontoppidan H. Mechanical aids to long expansion in non-intubated surgical patients. Am Rev Respir Dis 1980;122:109-19.

2 Celli BR, Rodriguez K, Snider GL. A controlled trial of intermittent positive pressure breathing, incentive spirometry and deep breathing exercise in preventing pulmonary complications after abdominal surgery. Am Rev Respir Dis 1984;130:12-5.

3 Kroenke K, Lawrence VA, Theroux JF, Tuley MR. Operative risk in patients with severe obstructive pulmonary disease. Arch Intern Med 1992;152:967-71.

4 Jackson C. Preoperative pulmonary evaluation. Arch Intern Med 1988;148:919-22.

5 Ford GT, Whitelaw W, Rosenal TW, Cruse PJ, Guenter CA. Diaphragmatic function after upper abdominal surgery in humans. Am Rev Respir Dis 1983;127:431-6.

6 Road JD, Burgess KR, Whitelaw WA, Ford GT. Diaphragm function and respiratory response after upper abdominal surgery in dogs. $\mathcal{F} A p p l$ Physiol 1984;57:576-82.

7 Wheatley RG, Somerville ID, Sapsford DJ, Jones JG. Postoperative hypoxemia: comparison of extradural, intramuscular and patient controlled opioid analgesia. $B r \mathcal{F}$ Anaesth 1990;64:267-75.

8 Esau SA. Effect of theophylline on membrane potential and contractile force in hamster diaphragm muscle in vitro. F Clin Invest 1986;77: 638-40.

9 Viires N, Aubier M, Murciano D, et al. Effects of theophylline on isolated diaphragmatic fibers. A model for pharmacologic studies on diaphrag-

10 Jansen JE, Sorensen AI, Naesh O, Erichsen CJ, Pedersen A. Effects of doxapram on postoperative pulmonary complications after upper abdominal surgery in high-risk patients. Lancet 1990;335:936-8. 